\title{
First-principles study of field-emission properties of nanoscale graphite ribbon arrays
}

\author{
S. F. Huang, ${ }^{1}$ T. C. Leung, ${ }^{1}$ Bin $\mathrm{Li}^{2}{ }^{2}$ and C. T. Chan ${ }^{2, *}$ \\ ${ }^{1}$ Department of Physics, National Chung Cheng University, Chia-Yi, Taiwan, Republic of China \\ ${ }^{2}$ Physics Department, Hong Kong University of Science and Technology, Clear Water Bay, Hong Kong, China
}

(Received 10 January 2005; published 18 July 2005)

\begin{abstract}
We applied a simple yet accurate self-consistent method using the density-functional formalism to study the field-emission properties of nanographite ribbons. We found some properties that are unique to nanostructure arrays, including the field-emission current being dominated by the contribution from off-zone-center $\pi$ states in some of the cases because of specific wave function symmetries, and an anomalous current density dependence on the inter-ribbons separation due to edge dipole effects. The applicability of Fowler-Nordheim analysis and some related problems are also discussed.
\end{abstract}

DOI: 10.1103/PhysRevB.72.035449

\section{INTRODUCTION}

In recent years, nanostructured materials, such as nanotubes ${ }^{1}$ and nanostructured arrays, ${ }^{2}$ have been shown to possess superior field-emission properties. For example, highly enhanced emission currents can be achieved for multiwalled carbon nanotubes. ${ }^{1}$ These properties are related to the special local environment on the nanometer scale and the electronic structures of nanostructures. Understanding the relationship between the local atomic configurations and electronic structures and the formation mechanism of the fieldemission current is important to further promote the realization of nanotube-based field-emission devices and to search for more nanostructures with potential applications in the field emission. The traditional Fowler-Nordheim (FN) theory ${ }^{3}$ and related formulas ${ }^{3,4}$ are constructed for freeelectron-like metals, and should not be expected to describe correctly the physics of nanostructures. That is why several methods for computing field-emission current have been introduced very recently. ${ }^{5-14}$ For example, two groups computed emission current using temporal wave function obtained by solving the time-dependent Schrödinger equation, and succeeded in explaining some field emission properties of the nanotubes and other related materials. ${ }^{8,9,13}$

As a simple prototypical system to explore the electronic structure of carbon-based nano-materials, nanographite ribbon had been studied by some theoretical and experimental groups for its special electronic structure properties in recent years. ${ }^{15-23}$ A kind of peculiar edge state localized at the edge carbon atoms of ribbon had been found near the Fermi level $\left(E_{F}\right)$ when the ribbon has a zigzag edge (denoted as the zigzag ribbon). ${ }^{15-17}$ Dangling-bond states exist in the ribbons without $\mathrm{H}$ atom termination, with their eigenenergies depending on the edge type (zigzag or armchair). ${ }^{20}$ In addition, some occupied states have C-C or C-H $\sigma$ characters, ${ }^{19}$ and others have the $\pi$ bond characters between $\mathrm{C}$ atoms. Many of these states may be related to the field-emission process. Tada et al. first studied the field-emission properties of the nanographite ribbons ${ }^{8,13}$ but they did not probe into the details of some of novel properties, especially for the ribbon having an armchair edge (denoted as the armchair ribbon). On the other hand, a previous study showed that the stacking manner of the zigzag ribbons array has important effect on the edge state. ${ }^{19}$ The dependence of the field-emission properties on the nanoscale structural parameters, such as the separation distance between the nanographite ribbon arrays, are also of interest. This system may also be viewed as a model for studying the field-emission properties of the mutilwalled carbon nanotubes. The nanographite ribbon can be also used to check the validity of applying the FN analysis method to the nanostructured systems.

In this paper, we adopt a simple $a b$ initio method within the local-density approximation (LDA) framework to calculate field-emission currents. A similar method has been proposed by Gohda et al. ${ }^{5,14}$ The advantage of this method is that it is very simple, yet the atomic and electronic structures are considered in full vigor within the LDA formulation under an explicit imposition of an external electric field $(E$ field), and it allows for a transparent interpretation of the field-emission properties based on the band structure. The method can be easily incorporated into existing LDA codes with minimal additional computation time. We will use this method to study some field-emission properties from the nanographite ribbons. It turns out that this system is an excellent prototypical system to illustrate some novel and yet subtle properties of the field emission from the nanostructures.

\section{METHOD AND PROCEDURE}

In the following, we will give a summary of our method. The plane-wave expansion of wave function and a supercell geometry are assumed. The system is periodic in the $x$ and $y$ directions.

We first solve the atomic and band structures explicitly within the LDA framework in the presence of an external $E$ field applied in the $z$ direction. The wave function for an eigenstate labeled by $\left(n, \vec{k}_{\|}\right)$(band and $\vec{k}$ point indices) can be written as

$$
\Psi_{n, \vec{k}_{\|}}(\vec{r})=\sum_{\vec{G}_{\|}} f_{n}\left(\vec{k}_{\|}+\vec{G}_{\|}, z\right) e^{i\left(\vec{k}_{\|}+\vec{G}_{\|}\right) \cdot \vec{r}_{\|}}
$$

In the vacuum region far enough from the slab, the $E$ field $F$ is essentially constant so that the potential 


$$
V(\vec{r}) \simeq V(z) \simeq-e F\left(z-z_{0}\right) .
$$

In this region, the wave function can be expanded in the linear combinations of $\Phi_{z}\left(\vec{K}_{\|}, z\right) e^{i \vec{K}_{\| \cdot} \cdot \vec{r}_{\|}}$terms $\left(\vec{K}_{\|}=\vec{k}_{\|}+\vec{G}_{\|}\right)$, where $\Phi_{z}\left(\vec{K}_{\|}, z\right)$ satisfies

$$
\left[\frac{\partial^{2}}{\partial z^{2}}+\frac{2 m e F}{\hbar^{2}}\left(z-z_{a}\right)\right] \Phi_{z}\left(\vec{K}_{\|}, z\right)=0,
$$

with $z_{a}$ being the classical turning point for the linear potential and being determined implicitly through $V\left(z_{a}\right)=\varepsilon_{n, \vec{k}_{\|}}$ $-\hbar^{2}\left|\vec{K}_{\|}\right|^{2} / 2 m$, where $\varepsilon_{n, \vec{k}_{\|}}$is the eigenenergy. The general solutions of $\Phi_{z}\left(\vec{K}_{\|}, z\right)$ are linear combinations of Airy functions. ${ }^{24}$ It can be deduced that

$$
\Phi_{z}\left(\vec{K}_{\|}, z\right)=C[\operatorname{Bi}(\xi)+i \operatorname{Ai}(\xi)],
$$

as long as it represents an outgoing wave in the fieldemission problem for $z>z_{a}$, where $C$ is a constant to be determined, $\xi=\left(2 m e F / \hbar^{2}\right)^{1 / 3}\left(z_{a}-z\right)$, and $\operatorname{Ai}(\xi)$ and $\operatorname{Bi}(\xi)$ are Airy functions.

In the vacuum region far away from the emitter so that the self-consistent potential $V(\vec{r})$ indeed approximates $-e F(z$ $-z_{0}$ ), we can choose a matching point $z=z_{m}$ at which

$$
f_{n}\left(\vec{k}_{\|}+\vec{G}_{\|}, z_{m}\right)=\Phi_{z}\left(\vec{K}_{\|}, z_{m}\right) .
$$

In addition, if the matching position $z_{m}$ is far enough away from the classical turning point $z_{a}$, the $\operatorname{Ai}(\xi)$ component in Eq. (4) is much smaller than the $\operatorname{Bi}(\xi)$ component. Then,

$$
C=\frac{f_{n}\left(\vec{k}_{\|}+\vec{G}_{\|}, z_{m}\right)}{\operatorname{Bi}\left[\left(\frac{2 m e F}{\hbar^{2}}\right)^{1 / 3}\left(z_{a}-z_{m}\right)\right]} .
$$

It is fairly straightforward to show that the current contribution from a $\vec{K}_{\|}=\vec{k}_{\|}+\vec{G}_{\|}$partial wave of the $n$th band is given by

$$
J_{n, \vec{k}_{\|}+\vec{G}_{\|}}=\frac{\hbar}{m} \operatorname{Im}\left[\Phi_{z}^{*}\left(\vec{K}_{\|}, z\right) \frac{\partial \Phi_{z}\left(\vec{K}_{\|}, z\right)}{\partial z}\right]=|C|^{2} \frac{\hbar}{\pi m}\left(\frac{2 m e F}{\hbar^{2}}\right)^{1 / 3} .
$$

The total current energy distribution (TED) can then be written as

$$
J(E)=2 \sum_{n, \vec{k}_{\|}} \delta\left(E-\varepsilon_{n, \vec{k}_{\|}}\right) \eta\left(\varepsilon_{n, \vec{k}_{\|}}\right) J_{n, \vec{k}_{\|}},
$$

and the total field-emission current density is

$$
J_{\text {total }}=\int J(E) d E
$$

where

$$
J_{n, \vec{k}_{\|}}=\sum_{\vec{G}_{\|}} \frac{\left|f_{n}\left(\vec{k}_{\|}+\vec{G}_{\|}, z_{m}\right)\right|^{2}}{\left|\operatorname{Bi}\left[\left(\frac{2 m e F}{\hbar^{2}}\right)^{\frac{1}{3}}\left(z_{a}-z_{m}\right)\right]\right|^{2}} \frac{\hbar}{\pi m}\left(\frac{2 m e F}{\hbar^{2}}\right)^{1 / 3},
$$

and $\eta\left(\varepsilon_{n, \vec{k}_{\|}}\right)$is Fermi-Dirac occupation function factor.

In this method, it is important to choose an appropriate matching point $\left(z_{m}\right)$, at which. (i) the potential should be

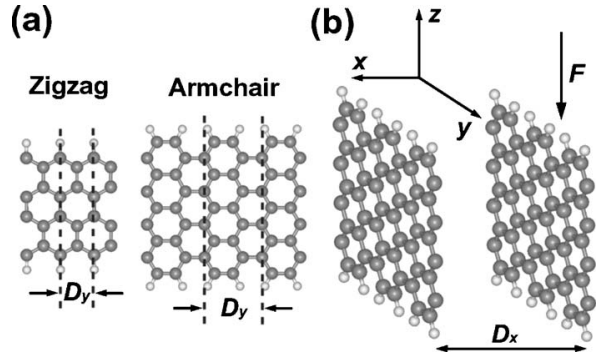

FIG. 1. (a) Structural schematics and (b) orientation of the unit cell for the nanographite ribbons with edges saturated by $\mathrm{H}$ atoms (open circles). The boundaries of primitive cells in the $y$ direction are shown using the dashed lines in (a), and the $E$ field $F$ is represented by an arrow in (b).

essentially linear (required for matching the wave function); (ii) the numerical noise in the wave function obtained from the first-principles calculations should be small enough; (iii) the $\operatorname{Ai}(\xi)$ component in Eq. (4) is much smaller than the $\operatorname{Bi}(\xi)$ component. The allowable value of $z_{m}$ is typically 6 to $8 \AA$ above the emitter surface. So this method demands that the vacuum layer be thick enough (generally $>20 \AA$ ) and has a maximum allowed $E$ field value depending on the system. The functions $f_{n}\left(\vec{k}_{\|}+\vec{G}_{\|}, z\right)$ are obtained by performing standard plane-wave pseudopotential calculations. We used the Vienna $a b$ initio simulation package (VASP), ${ }^{25-27}$ and the static $E$ field is imposed by putting a positive charge sheet in the vacuum on one side and a negative charge sheet on another side of the slab. ${ }^{28}$

Figure 1(a) shows the atomic structures of the nanographite ribbon arrays with two kinds of edges (zigzag and armchair), and the coordinate axes are defined in Fig. 1(b). The nanoribbon arrays comprise of graphitic strips that are infinitely long in the $y$ direction with primitive cells that measure 4.32 and $2.49 \AA$ for the armchair and zigzag ribbons, respectively. Five values of inter-ribbon distance $\left(D_{x}=5,10\right.$, $15,20,25 \AA)$ are considered. The $E$ field is imposed in the vertical $(z)$ direction [Fig. 1(b)], and the supercell has a vacuum thickness of $26 \AA$ in the $z$ direction. We use ultrasoft pseudopotentials ${ }^{29}$ and the generalized gradient approximation (GGA), ${ }^{30}$ with a plane-wave cutoff of $211 \mathrm{eV}$. The $\vec{k}$ points are sampled on a sufficiently dense grid in the twodimensional (2D) Brillouin zone (BZ), such as $(24 \times 28$ $\times 1)$ for the armchair ribbon. The atomic positions were relaxed until the magnitude of the forces became less than $0.1 \mathrm{eV} / \AA$, which also converged the total energy to within 1 meV. Our formulation allows the imposition of an $E$ field up to about $0.3 \mathrm{~V} / \AA$ for this system. We set the temperature for determining $\eta\left(\varepsilon_{n, \vec{k}_{\|}}\right)$to be $78 \mathrm{~K}$ and $z_{m}$ to be $7 \AA$ away from the ribbon edge. The qualitative features of the emission current are nearly independent of the temperature.

\section{RESULTS AND DISCUSSION}

\section{A. $\vec{k}$-resolved emission current and wave function symmetry}

Figure 2 shows the TED curves of the current emitted from four types of nanographite ribbons (pristine or $\mathrm{H}$ terminated with zigzag or armchair edge) under an $E$ field of 


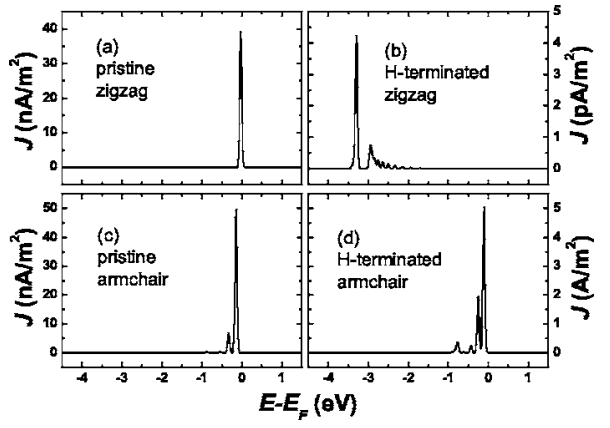

FIG. 2. TED curves of the emission current from four types of ribbons $\left(D_{x}=5 \AA, F=0.2 \mathrm{~V} / \AA\right.$ ) with $\vec{k}$ points being sampled in the full BZ for our calculations: (a) pristine zigzag; (b) H-terminated zigzag; (c) pristine armchair; (d) H-terminated armchair.

$0.2 \mathrm{~V} / \AA$. For the pristine and H-terminated zigzag ribbons, our results are indeed similar to the previous calculations. ${ }^{8}$ We note that the simulations of Tada et al. imposed a higher $E$ field of $1 \mathrm{~V} / \AA$ (such a high field is beyond our formalism), so the current magnitude will be different, but a qualitative comparison of the energy distribution should be possible. The results for two types of zigzag ribbons can be explained by examining their band structures, as discussed by Tada $e t$ $a l .{ }^{8}$ Figure 3 gives the band structures for four types of ribbons. There are flat dangling-bond bands crossing the $E_{F}$ near the BZ center for the pristine zigzag ribbon [Fig. 3(a)], giving rise to a strong field-emission current. These bands are removed when the edge is passivated with $\mathrm{H}$ atoms [Fig. 3(b)]. Although the H-terminated zigzag ribbon remains metallic, there is very small emission current near $E_{F}$ for the $\mathrm{H}$-terminated zigzag ribbon according to Eq. (10) since the states near $E_{F}$ (including the well-known edge states) have larger $\vec{k}_{\|}$and thus larger $z_{a}$ values.

The cases of armchair ribbons are more subtle. Our results show that the main peaks are all located near $E_{F}$ for the pristine and $\mathrm{H}$-terminated armchair ribbons, but in the results of Tada et al. these peaks are a few eV below $E_{F} \cdot{ }^{8}$ In these

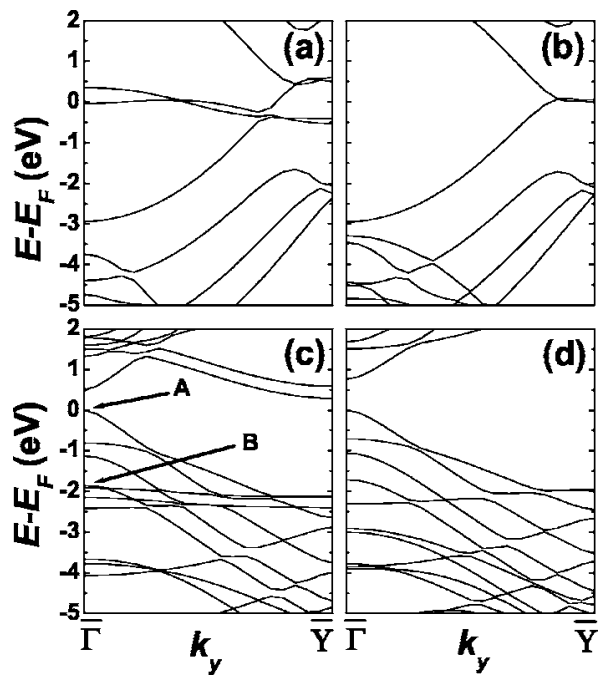

FIG. 3. Band structures of four types of ribbons with $D_{x}=5 \AA$ under an $E$ field of $F=0.2 \mathrm{~V} / \AA$ : pristine zigzag; (b) H-terminated zigzag; (c) pristine armchair; (d) H-terminated armchair.

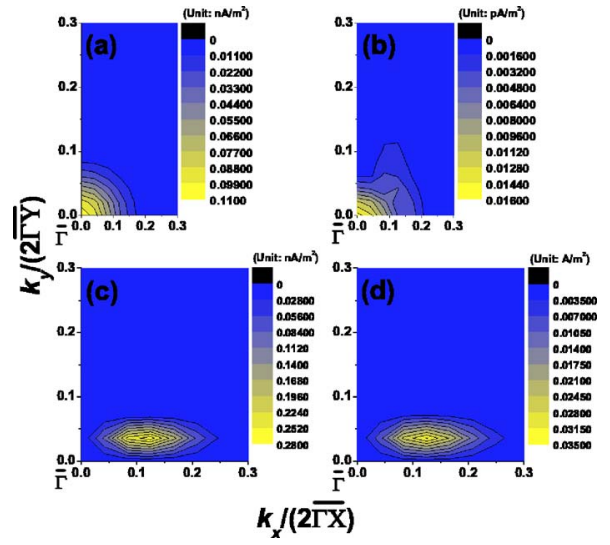

FIG. 4. (Color online) Contours of constant $\vec{k}_{\|}$-resolved emission current density in the 2D BZ for four types of ribbons with $D_{x}$ $=5 \AA$ under an $E$ field of $F=0.2 \mathrm{~V} / \AA$ : (a) pristine zigzag; (b) H-terminated zigzag; (c) pristine armchair; (d) H-terminated armchair.

two cases of armchair ribbons, the energy of the highest occupied state (or the valance band top) is defined as $E_{F}$, which is the same as the calculations of Tada et al. ${ }^{8}$ In fact, the energy gap near $E_{F}$ will decrease to zero in the limit of very large $z$ direction width of the armchair ribbon. ${ }^{15}$ From the band structures for two types of armchair ribbons [Figs. $3(\mathrm{c})$ and 3(d)], the highest occupied state is just at $\bar{\Gamma}$ point, so the small contribution of the highest occupied state and the states close to its energy to the emission current in the results of Tada et al. ${ }^{8}$ cannot be explained by the $\vec{k}_{\|}$effect as discussed in the case of $\mathrm{H}$-terminated zigzag ribbon.

The difference between the results of Tada et al. ${ }^{8}$ and ours for two types of armchair ribbons actually highlights the field-emission properties of nanostructures. In Fig. 4, we show the contours of $\vec{k}_{\|}$-resolved emission current in the 2D BZ for all four types of ribbons. We can see that most of the contribution to the total emission current comes from $\bar{\Gamma}$ point and its vicinity for the pristine and $\mathrm{H}$-terminated zigzag ribbons, but for the pristine and H-terminated armchair ribbons, most of the total current comes from a region that is off the high-symmetry lines [centered at the $\vec{k}_{\|}$point $(0.25 \overline{\Gamma X}$, $0.07 \overline{\Gamma Y})]$. We note that we sampled the full BZ, while the calculation of Tada et al. ${ }^{8}$ sampled $\vec{k}$ points only along the $\overline{\Gamma Y}$ line, which is basically fine for two types of zigzag ribbons, but missed much of the current contribution for the armchair ribbons. In fact, when we use the $\vec{k}$ points setting of $1 \times 28 \times 1$, i.e., only along $\overline{\Gamma Y}$, which is similar to the calculation of Tada et al. ${ }^{8}$ the main peaks in the TED curve for two types of armchair ribbons [Figs. 5(c) and 5(d)] are in the same energy range as in the results of Tada et al.. ${ }^{8}$

Equation (10) shows that for the occupied states, the $\vec{k}_{\mid-}$ and band-resolved emission current is the product of the factor $\left|f_{n}\left(\vec{k}_{\|}, z_{m}\right)\right|^{2}$ and a tunneling factor

$$
g\left(\varepsilon_{n, \vec{k}_{\|}}, \vec{k}_{\|}, z_{m}\right)=\frac{\frac{\hbar}{\pi m}\left(\frac{2 m e F}{\hbar^{2}}\right)^{1 / 3}}{\left|B i\left[\left(\frac{2 m e F}{\hbar^{2}}\right)^{1 / 3}\left(z_{a}-z_{m}\right)\right]\right|^{2}} .
$$

For simplicity, here we only consider the $\vec{G}_{\|}=0$ term which dominates the emission. Generally, $\bar{\Gamma}$ point emission is the 


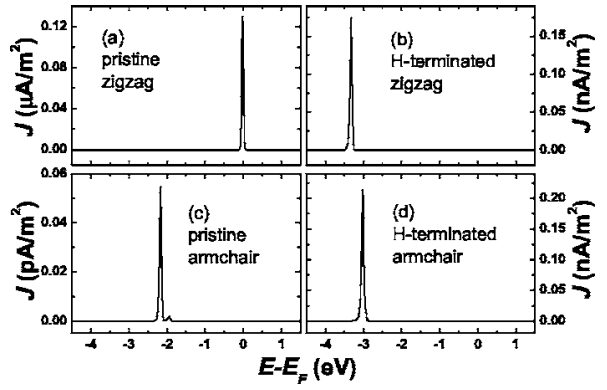

FIG. 5. TED curves of the emission current from four types of ribbons $\left(D_{x}=5 \AA, F=0.2 \mathrm{~V} / \AA\right)$ with $\vec{k}$ points being sampled only along $\overline{\Gamma Y}$ line in the BZ for our calculations: (a) pristine zigzag; (b) H-terminated zigzag; (c) pristine armchair; (d) H-terminated armchair.

strongest since $z_{a}$ is smallest at $\vec{k}_{\|}=0$ for the same energy, giving rise to a big tunneling factor $g\left(\varepsilon_{n, \vec{k}_{\|}}, \vec{k}_{\|}, z_{m}\right)$. This is just the $\vec{k}_{\|}$effect as mentioned before. As for the $\left|f_{n}\left(\vec{k}_{\|}, z_{m}\right)\right|^{2}$ factor, we can deduce

$$
\left|f_{n}\left(\vec{k}_{\|}, z_{m}\right)\right|^{2}=\left|<\Psi_{n, \vec{k}_{\|}}(\vec{r})\right| e^{i \vec{k}_{\|} \cdot \vec{r}_{\|}} \delta\left(z-z_{m}\right)>\left.\right|^{2}
$$

according to Eq. (1). Then,

$$
\left|f_{n}\left(\vec{k}_{\|}=0, z_{m}\right)\right|^{2}=\left|\iint_{S_{m}} \Psi_{n, \vec{k}_{\|}}(\vec{r}) d S\right|^{2},
$$

where $S_{m}$ is the $z=z_{m}$ plane. If an eigenstate at the $\bar{\Gamma}$ point $\left(\vec{k}_{\|}=0\right)$ has at least one nodal plane of the wave function perpendicular to the $x y$ plane (denoted as $m_{z} \neq 0$ state), the integral of its wave function on the $S_{m}$ plane will be zero due to the symmetry, and we will obtain a vanishing $\left|f_{n}\left(\vec{k}_{\|}, z_{m}\right)\right|^{2}$ factor. This kind of $\bar{\Gamma}$ state has no contribution to the emission current. For other $\bar{\Gamma}$ states, there exist the large lobes of the wave functions sticking out into the vacuum (denoted as $m_{z}=0$ states, similar to $s, p_{z}$ and $d_{z}^{2}$ orbitals), so the integrals of their wave functions on the $S_{m}$ plane, i.e., $\left|f_{n}\left(\vec{k}_{\|}, z_{m}\right)\right|^{2}$, are nonzero and these states will contribute to the emission current. Similar arguments about the effect of wave function symmetry on the field emission had been suggested in previous papers. ${ }^{12,31,32}$

The above $\bar{\Gamma}$ point symmetry selection rules are relaxed when we move to $\vec{k}_{\|} \neq 0$. So, something interesting happens. While the emission usually comes from the BZ center since the parallel momentum is the smallest, the $\vec{k}_{\|} \neq 0$ states on a band of which the $\bar{\Gamma}$ state is $m_{z} \neq 0$ state (denoted as $m_{z} \neq 0$ band) can contribute more to the field-emission current if it happens that all $\bar{\Gamma}$ states near $E_{F}$ are $m_{z} \neq 0$ states and inhibited by the symmetry. The chance of encountering such a situation is high in the cases of nanostructures because of the sparseness of their energy levels. For the armchair ribbon, this is precisely the case: the highest $m_{z}=0$ occupied states that can emit at the BZ center [indicated by "B" in Fig. 3(c)] are about $1.9 \mathrm{eV}$ below $E_{F}$, but there is a $m_{z} \neq 0$ band [of which the $\bar{\Gamma}$ state is indicated by "A" in Fig. 3(c)] near $E_{F}$. Figure 6(b) is the real part contour of wave function for the
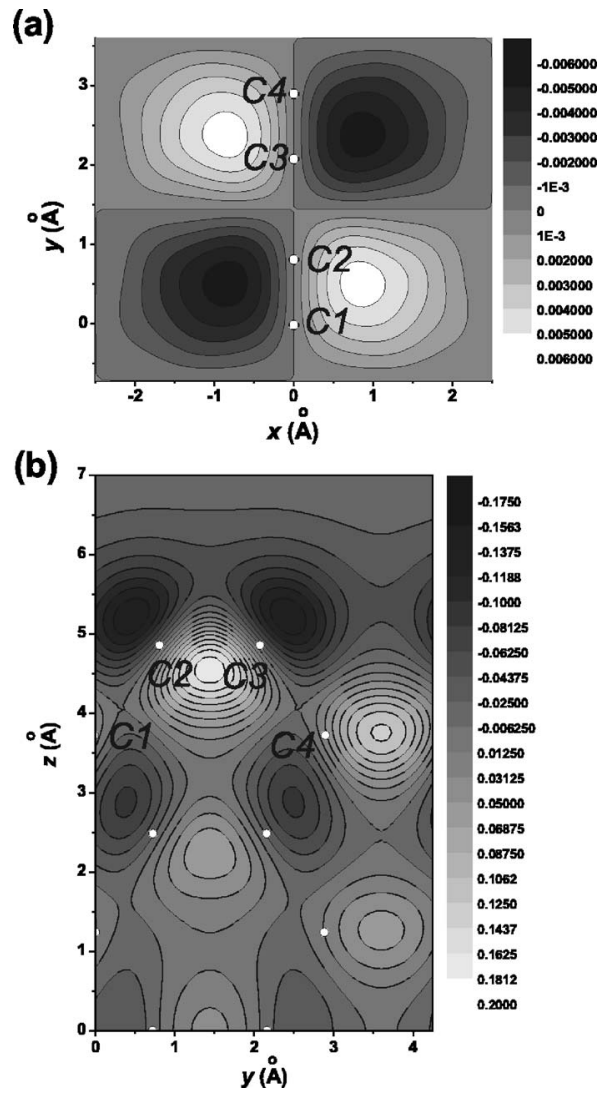

FIG. 6. (a) Contour of real part of the wave function for the state "A" [see Fig. 3(c)] in the plane $z=2.0 \AA$ away from the ribbon edge; (b) contour of real part of the wave function for the state "B" [see Fig. 3(c)] in the plane of the ribbon strip. The open circles indicate the positions of the carbon atoms in-cluding four atoms at the pristine armchair ribbon edge: "C1," "C2," "C3," and "C4."

state "B" in the plane of the ribbon strip, showing that this state is of the dangling bond state type $\mathrm{e}^{20}$ and has $\sigma$ bond character between the edge carbon atoms $\mathrm{C}_{2}$ and $\mathrm{C}_{3}$. The state $\mathrm{A}$ is of $\pi^{*}$ bond originating from the $p_{x}$ orbital of carbon atoms, and the real part contour of its wave function in the plane $z=2.0 \AA$ away from the ribbon edge is shown in Fig. 6(a). So, the following two effects are in competition: the emitting states should have energies as close to $E_{F}$ as possible, but at the same time they should be allowed by the symmetry to emit. As a compromise, the off-zone-center states on that $m_{z} \neq 0$ band near $E_{F}$ contribute to most of the field emission current. The same mechanism works in the case of the H-terminated armchair ribbon. The emission current from those $m_{z}=0$ occupied states including the dangling bond state $\mathrm{B}$ still exists in the TED curve, but the magnitude is much smaller [see Figs. 5(c) and 5(d)] because of their lower energies, and so the corresponding peaks do not show up conspicuously in the TED curves when $\vec{k}$ points is properly sampled in the full BZ [Figs. 2(c) and 2(d)]. This peculiar behavior of the emission from the armchair ribbons is not because of the inter-ribbon interaction. The TED of the emission current is qualitatively similar for different interribbon distances $D_{x}(\geqslant 5 \AA)$ as long as the full BZ is sampled. 


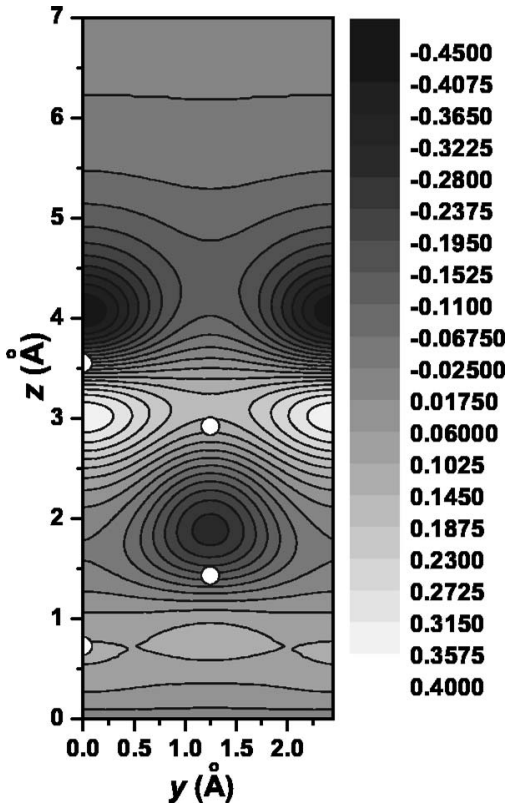

FIG. 7. Contour of real part of the wave function for the dangling bond state at $E_{F}$ and $\bar{\Gamma}$ point of the pristine zigzag ribbon in the plane of the ribbon strip. The positions of the carbon atoms was indicated by using the open circles.

As for the cases of two types of zigzag ribbons, the states contributing to most of the emission current are $m_{z}=0$ states. For example, the dangling bond state near $E_{F}$ and at $\bar{\Gamma}$ point of the pristine zigzag ribbon originates from the $p_{z}$ orbitals of the edge carbon atoms. Figure 7 shows the real part contour of its wave function in the plane of the ribbon strip. So most of the contribution to the total emission current comes from the $\bar{\Gamma}$ point and its vicinity for two types of zigzag ribbons.

\section{B. Geometrical and other related factors}

For an array of nanostructures, their separation will affect field-emission properties, at least for the reason of the wellknown field enhancement effect. As for the system of a nanographite ribbons array, the inter-ribbon (lateral) distance $D_{x}$ is an important structural parameter for determining the efficiency of field-emission. Figure 8 gives total field-emission current density values $J_{\text {total }}$ for different inter-ribbon distances $D_{x}$ in an $E$ field of $0.2 \mathrm{~V} / \AA$. We find that for the pristine ribbons, the emission current densities $J_{\text {total }}$ increase when the inter-ribbon distance $D_{x}$ is enlarged, but for the H-terminated ribbons, the current densities $J_{\text {total }}$ initially decrease and finally increase when $D_{x}$ is enlarged. It should be noted the above behaviors are independent on whether the ribbon edge is zigzag or armchair. Such a behavior is rather anomalous, since the geometrical field enhancement effect is not dependent on the edge structure of the ribbon and should result in the same trend of the emission current for all types of ribbons. The inter-ribbon interaction should be very small when $D_{x} \geqslant 5 \AA$, so the observed changes are not induced by the band structure effect.

The FN formulas ${ }^{3}$ show that the emission current depends on the work function of the emitter surface very sensitively.

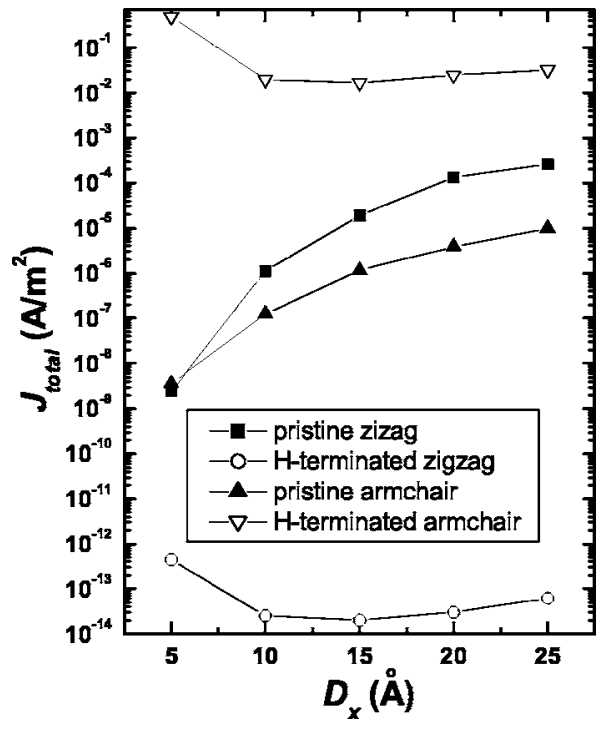

FIG. 8. The total field-emission current density for four types of ribbons with different inter-ribbons distances $D_{x}$ under an $E$ field of $F=0.2 \mathrm{~V} / \AA$.

This suggests that the anomalous behaviors of $J_{\text {total }}-D_{x}$ plots may be related to the special dependence of the work function on the inter-ribbon distance $D_{x}$. Through explicit calculations we found that when the ribbon strips are closely spaced, the work function of the system actually depends on $D_{x}$. Figure 9 (a) presents the work functions $\phi_{\mathrm{GGA}}$ of four types of ribbons with different $D_{x}$ by our GGA calculations. We find that when $D_{x}$ is not too large, the dependence of the emission current density $J_{\text {total }}$ on the inter-ribbon distance $D_{x}$ mainly originates from a special relationship between the work function $\phi_{\mathrm{GGA}}$ and $D_{x}$, and this phenomena is due to an edge dipole effect. It is rather unique in an array of nanostructures, and can be explained as follows. According to Wigner and Bardeen's theory, ${ }^{33}$ the work function of a material surface can be written as

$$
\phi=-\mu-\frac{e P}{\varepsilon_{0} A},
$$

where $\mu$ is the chemical potential in the interior of the material, $P$ and $A$ are, respectively, the surface dipole moment and the area per surface primitive cell. For the nanographite ribbons arrays, Eq. (14) becomes

$$
\phi=-\mu-\frac{e P_{e}}{\varepsilon_{0} D_{x} D_{y}},
$$

where $P_{e}$ is the edge dipole moment per $x y$-plane primitive cell of one ribbon strip, and $D_{y}$ is the length of the primitive cell along the $y$ direction, i.e., along the ribbon strip edge direction [see Fig. 1(a)]. $P_{e}$ is essentially determined by the electronic properties of a single ribbon, and is almost constant when $D_{x}$ is large enough $\left(D_{x} \geqslant 5 \AA\right)$. For the pristine nanographite ribbons, the dangling bond states result in a negative edge dipole moment (pointing into the ribbon), causing $\phi$ to decrease with increasing $D_{x}$. Figures 10 (a) and 10(b) show the local Coulomb potentials, that are averaged 

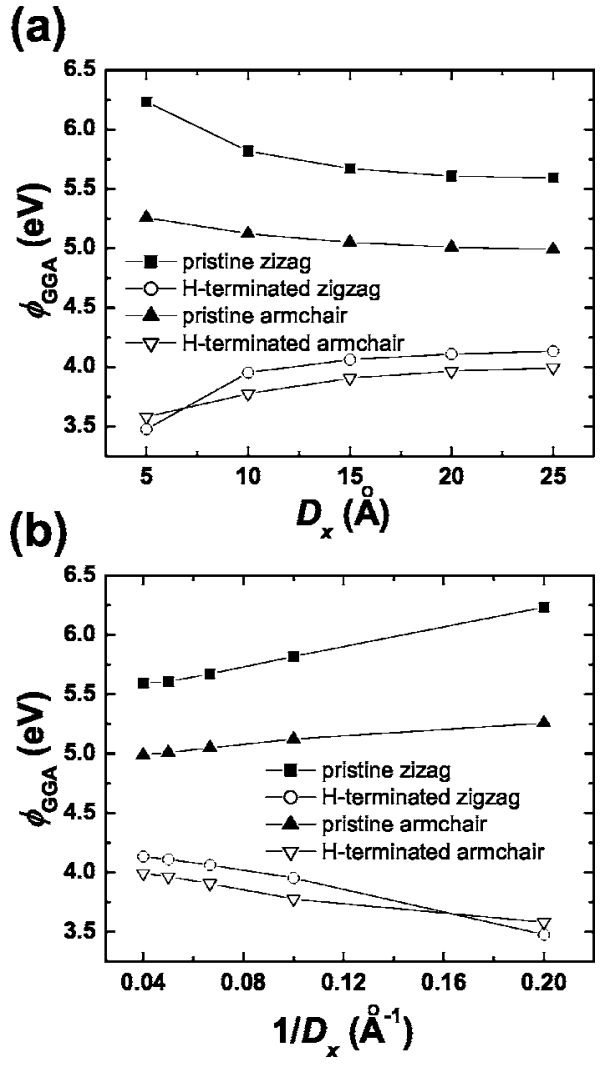

FIG. 9. (a) Calculated work function $\phi_{\mathrm{GGA}}$ for four types of ribbons with different inter-ribbons distances $D_{x}$ and (b) plots for $\phi_{\mathrm{GGA}}$ versus $1 / D_{x}$.

in the $y$ direction with $x$ coordinates fixed in the middle plane between the ribbon strips and in the plane of the ribbon strip, along the $z$ direction for the pristine zigzag and armchair ribbons with $D_{x}=5 \AA$ in zero $E$ field. One can see that for the $y$-averaged potential in between two ribbon strips [solid lines in Figs. 10(a) and 10(b)], it increases from the "interior" to the vacuum region, which is an obvious evidence of the negative edge dipole moment for the pristine ribbons. For the $\mathrm{H}$-terminated nanographite ribbons, the edge dipole moment is positive (pointing out) because the dangling bonds are saturated and the electrons are mainly localized between carbon and hydrogen atoms, so $\phi$ increases when $D_{x}$ is increased. Figures 10(c) and 10(d) present evidence of the positive edge dipole moments for the $\mathrm{H}$-terminated zigzag and armchair ribbons. In Fig. 9(b), the plots of $\phi_{\mathrm{GGA}}$ versus $1 / D_{x}$ obtained from our GGA calculations also suggest a dependence of work function on the $D_{x}$ similar to Eq. (15). Such a change of the work function due to the change of edge dipole density $P_{e} /\left(D_{x} D_{y}\right)$ causes the initial increase (decrease) of the emission current from the pristine $(\mathrm{H}-$ terminated) ribbon according to the FN formulas. ${ }^{3}$

Of course, the classical field enhancement effect still exists in the cases of these ribbons. As a geometrical effect, it always enhances the field emission current density when $D_{x}$ is enlarged since the local $E$ field near the ribbon edge is always higher for the larger $D_{x}$. The effect due to the dipole density and field enhancement reinforces each other for the cases of the pristine ribbon, so the total emission current

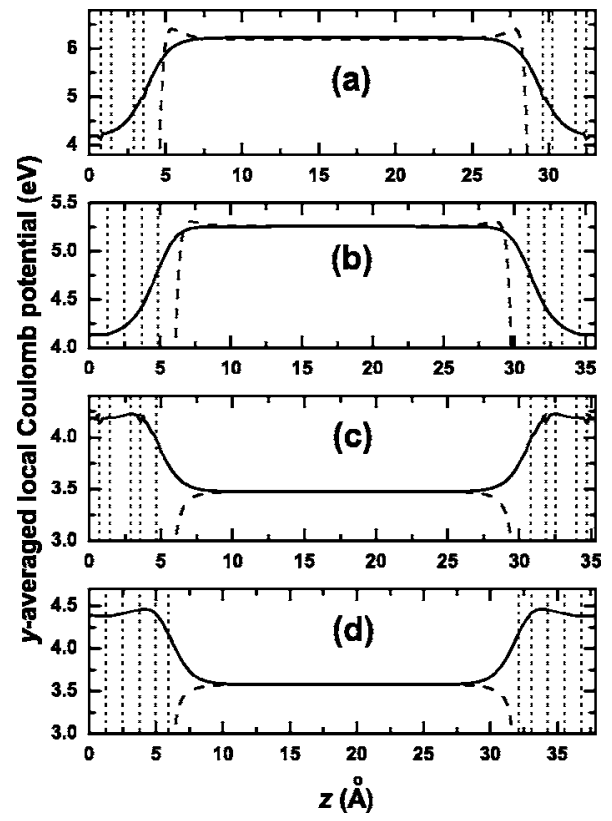

FIG. 10. The $y$-averaged local Coulomb potentials with $x$ coordinates fixed in the middle plane between the ribbon strips (solid line) and in the plane of the ribbon strip (dashed line) along the $z$ direction for four types of ribbons $\left(D_{x}=5 \AA, F=0\right)$ : (a) pristine zigzag; (b) pristine armchair; (c) H-terminated zigzag; (d) $\mathrm{H}$-terminated armchair. The $z$-coordinates of all atoms are indicated by using the dot lines.

densities $J_{\text {total }}$ always increase when $D_{x}$ is enlarged. But in the cases of $\mathrm{H}$-terminated ribbons, these two effects are opposite. When the inter-ribbons distance $D_{x}$ is large enough, the decreases of emission current densities induced by the edge dipole density effect turns to be slower because $1 / D_{x}$ has been close to zero. So the field enhancement effect begin to determine the trend of emission current density $J_{\text {total }}$ with increasing $D_{x}$, as shown in Fig. 8 .

\section{The applicability of Fowler-Nordheim analysis and related problems}

FN analyses are frequently applied to the field emission studies for the nanotubes and other nanostructures. An important purpose of the $\mathrm{FN}$ analyses is to obtain the work function of the emitter surface. We examine here whether the work function value obtained from the $\mathrm{FN}$ analyses is the same as the real work function of the nanostructure system.

Figure 11 shows FN plots for four types of ribbons with the inter-ribbon distance $D_{x}=5 \AA$. One can see that all lines are rather linear up to $0.3 \mathrm{~V} / \AA$. In Table I, the work functions deduced from the $\mathrm{FN}$ formula ${ }^{3}$

$$
J_{\text {total }} \propto F^{2} e^{-(4 \sqrt{2 m} / 3 \hbar e) \phi^{3 / 2} F^{-1}},
$$

and also the image-potential corrected $\mathrm{FN}$ formula ${ }^{4}$ are compared with the work functions obtained directly by our GGA calculations. We can find poor agreement for the case of $\mathrm{H}$-terminated zigzag ribbon. The FN theory assumes that the emitter is of free-electron-like type, and the most of emission current comes from the states near $E_{F}$. But in the case of the 


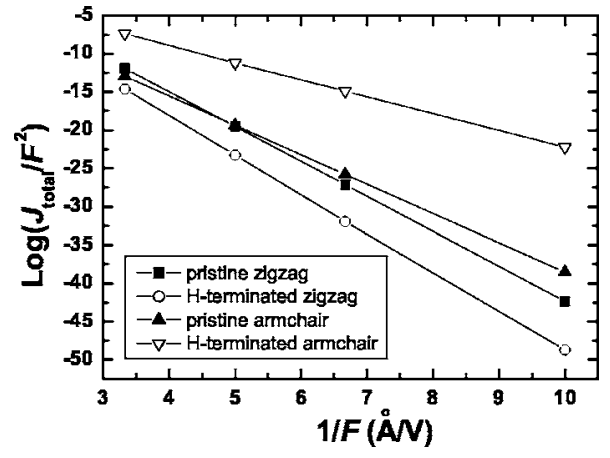

FIG. 11. The FN plots of the total emission current density per the primitive cell along the ribbon edge (unit: $\mu \mathrm{A} / \mathrm{nm}$ ) for four types of ribbons with $D_{x}=5 \AA$.

$\mathrm{H}$-terminated zigzag ribbon, the states at about $3 \mathrm{eV}$ below $E_{F}$ contribute to the most of the emission current. The work function obtained from the FN plot for the H-terminated zigzag ribbon actually reflects the energy difference between the vacuum level and those states contributing to the most of the current. For two cases of the armchair ribbons, we find that the work functions deduced from the FN formula fitting are about 5-7 \% larger than those calculated by the GGA. This departure can also be understood by considering that the eigenstates contributing to the most of the current are at about $0.2-0.4 \mathrm{eV}$ below $E_{F}$, and that these states are not at $\bar{\Gamma}$ point. In fact, the work function deduced from the FN plot can be regarded as an "effective" work function

$$
\phi_{\text {eff }}=\phi+E_{F}-\varepsilon_{n, \vec{k}_{\|}}^{m}+\frac{\hbar^{2}\left|\vec{k}_{\|}^{m}\right|^{2}}{2 m},
$$

where $\varepsilon_{n, \vec{k}_{\|}}^{m}$ and $\vec{k}_{\|}^{m}$ are the eigenenergies and $\vec{k}$ point indices of the states contributing to the most of the current. This simple modification may make FN formula consistent with the field emission experimental results of nanostructures for the weak to moderate $E$ fields. In addition, we should not assume that the FN plot still remains linear under the higher $E$ field as it is always dangerous to extrapolate.

We also noted that the edge dipole moment density is positive (negative) for the H-terminated (pristine) ribbon, causing the H-terminated ribbons to have much lower work functions (see Table I). Similar results had also been obtained by the calculations of Ramprasad et al. ${ }^{34}$ This accounts for the much higher emission current density from the

TABLE I. Work functions (units of eV) of four types of ribbon arrays with $D_{x}=5 \AA$ : $\phi_{\mathrm{FN}}$ (deduced from the FN formula), $\phi_{\mathrm{FN}-\mathrm{IP}}$ (deduced from the image-potential corrected FN formula), and $\phi_{\mathrm{GGA}}$ (calculated directly by the vASP using the GGA).

\begin{tabular}{cccc}
\hline \hline & $\phi_{\mathrm{FN}}$ & $\phi_{\mathrm{FN}-\mathrm{IP}}$ & $\phi_{\mathrm{GGA}}$ \\
\hline Pristine zigzag & 6.19 & 6.24 & 6.23 \\
H-terminated zigzag & 6.67 & 6.72 & 3.48 \\
Pristine armchair & 5.51 & 5.57 & 5.26 \\
H-terminated armchair & 3.83 & 3.92 & 3.58 \\
\hline \hline
\end{tabular}

H-terminated armchair ribbon than that from the pristine armchair ribbon (see Figs. 8 and 11). However, the emission current from the pristine zigzag ribbon turns out to be much higher than that from the H-terminated zigzag ribbon, even though the latter has a lower work function. That is because there happens to be a dangling bond band right near $E_{F}$ for the pristine zigzag ribbon, which was removed by the $\mathrm{H}$-saturation for the H-terminated zigzag ribbon. This also causes the "effective" work function of the H-terminated zigzag to be apparently larger than its actual work function as mentioned above.

From Fig. 11, we can see that the emission current density from the pristine zigzag ribbon is lower than that from the pristine armchair ribbon when the external $E$ field $F$ $\leqslant 0.2 \mathrm{~V} / \AA$, consistent with the fact that the work function of the former is higher than that of the latter; but when the $E$ field is high enough (such as $0.3 \mathrm{~V} / \AA$ ), the emission current density from the pristine zigzag ribbon is higher than that from the pristine armchair ribbon. As discussed before, the emission current is determined jointly by two factors: the factor $\left|f_{n}\left(\vec{k}_{\|}, z_{m}\right)\right|^{2}$ and the tunneling factor $g\left(\varepsilon_{n, \vec{k}_{\|}}, \vec{k}_{\|}, z_{m}\right)$. The tunneling factor brings a specific relationship between the emission current and the external $E$ field, and this relationship makes the FN plot for the material with a higher work function have a larger slope [see Eq. (16)], i.e., the current increases more rapidly when the $E$ field is enhanced. The factor $\left|f_{n}\left(\vec{k}_{\|}, z_{m}\right)\right|^{2}$ is related to the properties of wave functions of the states contributing to the most of emission current as discussed before. Considering the fact that the most of emission currents from the pristine zigzag and armchair ribbons are from the $m_{z}=0$ dangling bond states at $E_{F}$ and the $m_{z} \neq 0 \mathrm{C}-\mathrm{C} \pi^{*}$ bond band near $E_{F}$ respectively, we can deduce that the $\left|f_{n}\left(\vec{k}_{\|}, z_{m}\right)\right|^{2}$ factor of the former is larger than that of the latter. Since the pristine zigzag ribbon has the larger slope of FN plot and $\left|f_{n}\left(\vec{k}_{\|}, z_{m}\right)\right|^{2}$ factor than the pristine armchair ribbon, the emission current from the former can exceed that from the latter when the $E$ field is larger than some value, as shown in Fig. 11.

\section{SUMMARY}

We used a first-principles method to study the field emission from the nanographite ribbon arrays. We found that the field emission properties of these nanostructures are complex and subtle, and the energy distribution and intensity of the emission current are the result of the competition of many factors. These factors include band structure effect, wave function symmetry, edge dipole effect and field enchancement effect. The calculations for the nanoribbon serves to illustrate these points.

\section{ACKNOWLEDGMENTS}

We thank Professor J. G. Che for valuable discussions. This work was supported by Hong Kong RGC through Grant No. HKUST6152/01P. S.F.H. and T.C.L. were supported by National Science Council Grant No. NSC 92-2112-M-194018, and a grant of computer time at the National Center for High Performance Computing. 
*Electronic address: phchan@ust.hk

${ }^{1}$ See, e.g., A. G. Rinzler, J. H. Hafner, P. Nikolaev, L. Lou, S. G. Kim, D. Tomanek, P. Nordlander, D. T. Colbert, and R. E. Smalley, Science 269, 1550 (1995).

${ }^{2}$ See, e.g., S. Fan, M. G. Chapline, N. R. Franklin, T. W. Tombler, A. M. Cassell, and H. Dai, Science 283, 512 (1999).

${ }^{3}$ R. H. Fowler and L. Nordheim, Proc. R. Soc. London, Ser. A 119, 173 (1928).

${ }^{4}$ See, e.g., C. D. Ehrlich and E. W. Plummer, Phys. Rev. B 18, 3767 (1978).

${ }^{5}$ Y. Gohda, Y. Nakamura, K. Watanabe, and S. Watanabe, Phys. Rev. Lett. 85, 1750 (2000).

${ }^{6}$ Ch. Adessi and M. Devel, Phys. Rev. B 62, R13314 (2000).

${ }^{7}$ R. Ramprasad, L. R. C. Fonseca, and Paul von Allmen, Phys. Rev. B 62, 5216 (2000).

${ }^{8}$ K. Tada and K. Watanabe, Phys. Rev. Lett. 88, 127601 (2002).

${ }^{9}$ S. Han, M. H. Lee, and J. Ihm, Phys. Rev. B 65, 085405 (2002).

${ }^{10}$ T. Ohwaki, H. Ishida, and A. Liebsch, Phys. Rev. B 68, 155422 (2003).

${ }^{11}$ H. Ishida, D. Wortmann, and T. Ohwaki, Phys. Rev. B 70, 085409 (2004).

${ }^{12}$ M. J. G. Lee and Z. A. Ibrahim, Phys. Rev. B 70, 125430 (2004).

${ }^{13}$ M. Araidai, Y. Nakamura, and K. Watanabe, Phys. Rev. B 70, 245410 (2004).

${ }^{14}$ Y. Gohda and S. Watanabe, J. Phys.: Condens. Matter 16, 4685 (2004).

${ }^{15}$ K. Nakada, M. Fujita, G. Dresselhaus, and M. S. Dresselhaus, Phys. Rev. B 54, 17954 (1996).

${ }^{16}$ M. Fujita, K. Wakabayashi, K. Nakada, and K. Kusakabe, J. Phys. Soc. Jpn. 65, 1920 (1996).
${ }^{17}$ O. E. Andersson, B. L. V. Prasad, H. Sato, T. Enoki, Y. Hishiyama, Y. Kaburagi, M. Yoshikawa, and S. Bandow, Phys. Rev. B 58, 16387 (1998).

${ }^{18}$ K. Wakabayashi, M. Fujita, H. Ajiki, and M. Sigrist, Phys. Rev. B 59, 8271 (1999).

${ }^{19}$ Y. Miyamoto, K. Nakada, and M. Fujita, Phys. Rev. B 59, 9858 (1999).

${ }^{20}$ T. Kawai, Y. Miyamoto, O. Sugino, and Y. Koga, Phys. Rev. B 62, R16349 (2000).

${ }^{21}$ Y.-L. Lee and Y.-W. Lee, Phys. Rev. B 66, 245402 (2002).

${ }^{22}$ K. Kusakabe and M. Maruyama, Phys. Rev. B 67, 092406 (2003).

${ }^{23}$ T. Hikihara, X. Hu, H.-H. Lin, and C.-Y. Mou, Phys. Rev. B 68, 035432 (2003).

${ }^{24}$ J.-N. Chazalviel and Y. Yafet, Phys. Rev. B 15, 1062 (1977).

${ }^{25}$ G. Kresse and J. Hafner, Phys. Rev. B 47, R558 (1993).

${ }^{26}$ G. Kresse and J. Hafner, Phys. Rev. B 49, 14251 (1994).

${ }^{27}$ G. Kresse and J. Furthmüller, Comput. Mater. Sci. 6, 15 (1996).

${ }^{28}$ See, e.g., J. Neugebauer and M. Scheffler, Surf. Sci. 287-288, 572 (1993).

${ }^{29}$ G. Kresse and J. Hafner, J. Phys.: Condens. Matter 6, 8245 (1994).

${ }^{30}$ J. P. Perdew and Y. Wang, Phys. Rev. B 45, 13244 (1992).

${ }^{31}$ J. W. Gadzuk and E. W. Plummer, Rev. Mod. Phys. 45, 487 (1973).

${ }^{32}$ S. Han and J. Ihm, Phys. Rev. B 66, 241402 (2002).

${ }^{33}$ E. Wigner and J. Bardeen, Phys. Rev. 48, 84 (1935).

${ }^{34}$ R. Ramprasad, P. von Allmen, and L. R. C. Fonseca, Phys. Rev. B 60, 6023 (1999). 